\title{
M-QAM BER Analysis of ZF MIMO Transmissions with Stochastic Interference Model for LTE
}

\author{
Albert Mráz*, László Pap \\ Department of Networked Systems and Services, Budapest University of Technology and Economics (BME), \\ H-1117 Budapest, Hungary. \\ * Corresponding author. Tel.: +36 1463 3227; email: mraz@hit.bme.hu \\ Manuscript submitted September 10, 2014; accepted April 14, 2015. \\ doi: 10.17706/ijcce.2015.4.5.309-317
}

\begin{abstract}
This work provides an exact analytical method for the calculation of the bit error rate for MIMO-ZF transmission, assuming arbitrary $M$ level quadrature amplitude modulation, to substitute the existing approximating solutions in the literature in the presence of additive white Gaussian noise. The authors have extended the calculations with a 3GPP LTE specified spatial correlation model in order to get realistic performance results and a usable tool in a realistic standardized mobile system. In addition, the bit error rate calculation method has been completed by a stochastic interference model, which influences the received interference energy by several different parameters, which will be assumed as random variables in this work. The interference model can be considered flexible in the sense that different random distributions can be defined for the implied parameters. In this work, the authors provide analytic bit error rate calculation results applying the mentioned stochastic interference model according to a frequency hopping scenario.
\end{abstract}

Key words: BER, MIMO, stochastic interference model, zero forcing.

\section{Introduction}

Multiple-input multiple-output (MIMO) transmission represents a current topic within the research activities of advanced wireless networks [1]. Several works deal with the bit error rate (BER) analysis of MIMO schemes, applying $M$ level QAM (quadrature amplitude modulation). In [2] an adaptive soft parallel interference canceller (ASPIC) is proposed for turbo coded MIMO, considering maximum ratio diversity combining (MRC) diversity. [3] provides an union bound estimation for the BER at high signal-to-noise-ratio (SNR) domains, without considering the symbol mapping. The authors of [4] give BER calculation for orthogonal space-time block codes (OSTBCs), [5] investigates the BER of linear minimum mean-square error (LMMSE) receiver with. In [6] analytical expressions have been given for the BER of convolutional coded MIMO system for high SNR domain. [7] provides calculations for the case of bit-loaded orthogonal frequency-division multiplexing (OFDM).

The goal of this paper is to provide a general solution for the BER calculation of MIMO, considering arbitrary $M$ level of QAM, including the effects gray symbol mapping, and applying a stochastic interference model, by considering zero forcing (ZF) receiver. In addition, we introduce a spatial correlation model defined in the LTE specification and extend it to arbitrary MIMO sizes, to investigate our BER calculations in an existing system.

This paper is organized as follows: Section 2 contains the model of the MIMO ZF transmission. Section 3 
shows an $M$-QAM BER calculation method for average interference level and considering the effect of Additive White Gaussian Noise (AWGN). In Section 4 we provide a spatial correlation model, Section 5 contains the extension of the interference model by random variables (RVs). Calculation results are illustrated in Section 5.

\section{System Model}

This section contains the mathematical framework of the MIMO ZF transmission, which will be the base of the BER calculations, considering a stochastic interference model.

\subsection{The MIMO Model}

Based on the system description in [8], let us denote the number of transmitter- (TX) and receiver (RX) antennas with $N_{\mathrm{T}}$ and $N_{\mathrm{R}}$ respectively. At the TX side, complex data symbols are grouped into an $N_{\mathrm{T}} \times 1$ sized $\mathbf{x}$ vector with

$$
\mathbf{x}=\mathbf{P}^{\frac{1}{2}} \mathbf{V} \mathbf{s}
$$

with $\mathbf{s}$ being an $N_{\mathrm{T}} \times 1$ vector with unit energy $M$-QAM symbols, and $\mathbf{V}$ a $N_{\mathrm{T}} \times N_{\mathrm{T}}$ unitary linear so called precoding matrix. In addition, $\mathbf{P}=\operatorname{diag}\left(p_{1}, \ldots, p_{n}, \ldots, p_{N_{\mathrm{T}}}\right)$, with $n \in\left(1,2, \ldots, N_{\mathrm{T}}\right)$, in which $p_{n}=\phi_{n} \frac{N_{\mathrm{R}}}{N_{\mathrm{T}}}$ with

$$
\phi=N_{\mathrm{R}} \frac{\mathbb{E}\left[\mathbf{x}_{\mathrm{T}}^{\dagger} \mathbf{x}_{\mathrm{T}}\right]}{\mathbb{E}\left[\mathbf{n}^{\dagger} \mathbf{n}\right]}
$$

TX side SNR values for stream $n$. The received signal vector after affected by the MIMO channel and the AWGN can be expressed with

$$
\mathbf{y}=\mathbf{H}_{w} \mathbf{R}_{\mathrm{T}}^{\frac{1}{2}} \mathbf{x}+\mathbf{n},
$$

where $\mathbf{H}_{w}$ is defined as an $N_{\mathrm{R}} \times N_{\mathrm{T}}$ matrix with independent and identically distributed (i.i.d.) complex gaussian entries with $\widetilde{N}(0,1)$. The TX side $N_{\mathrm{R}} \times N_{\mathrm{T}}$ correlation matrix is denoted by $\mathbf{R}_{\mathrm{T}}$. Let us denote $\mathbf{H}=\mathbf{H}_{w} \mathbf{R}_{\mathrm{T}}^{\frac{1}{2}}$ as an effective $N_{\mathrm{R}} \times N_{\mathrm{T}}$ MIMO channel matrix with correlated entries. After applying the MIMO detection algorithm, the estimated complex signal vector can be expressed as $\mathbf{z}=\mathbf{G y}$, where $\mathbf{G}=\left(\mathbf{H P}^{\frac{1}{2}} \mathbf{V}\right)^{\dagger}$ represents the application of the ZF receiver, and $(.)^{\dagger}$ denotes the pseudo-inverse of a matrix.

\subsection{SINR Distribution in Case of MMSE MIMO Reception}

It has been shown in [9] that the behavior of $\gamma_{n}$ for stream $n$ can be approximated by Gamma distribution with the probability distribution function (pdf) of

$$
f_{\mathrm{SINR}_{n}}(\gamma)=\frac{\gamma^{\alpha-1} e^{-\gamma / \theta}}{\Gamma(\alpha) \theta^{\alpha}}
$$


where $\alpha=N_{\mathrm{R}}-N_{\mathrm{T}}+1$ and $\theta=\frac{1 / N_{\mathrm{R}}}{\left[\mathbf{R}^{-1}\right]_{n, n}}$, in which $[\cdot]_{n, n}$ indicates the $(n, n)$-th entry of a matrix. In addition

$$
\mathbf{R}=\phi \frac{N_{\mathrm{R}}}{N_{\mathrm{T}}} \mathbf{P}^{\frac{1}{2}} \mathbf{V} \mathbf{R}_{\mathrm{T}} \mathbf{V}^{\mathrm{H}} \mathbf{P}^{\frac{1}{2}}
$$

i.e. $\theta$ has been derived from the generalized covariance matrix as it can be found in [9]. That is, $\theta$ will 'mediate' the effects of the correlated channel model on the observable BER. We will assume $\theta=\theta_{n}$ and $\alpha=\alpha_{n}$. For $\mathbf{V}=\mathbf{I}_{N_{\mathrm{T}}}$ we get the simple expression $\theta=C_{\text {corr }} \cdot \phi$, with a $C_{\text {corr }}$ correlation constant (Table 1).

\section{BER Calculation for MIMO with ZF}

In the following, we provide more accurate BER expressions for $M$-QAM ZF compared to [8], by considering the symbol mapping method.

\subsection{BER Framework for M-QAM with Gray Coding and AWGN}

An exact and general BER calculation has been provided in [10] for AWGN and Gray mapping. The proposed method calculates $P_{\mathrm{b}}(\gamma, l)$ values at first, expressed with

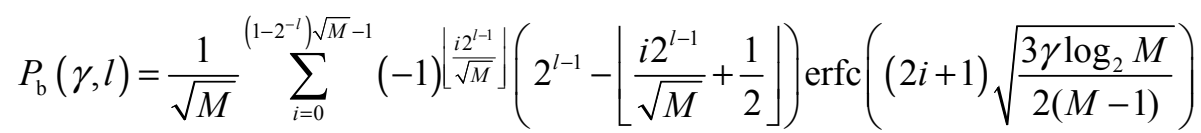

For the $l$-th bit of a symbol, where $l \in\left\{1,2, \ldots, \log _{2}(M)\right\}, \gamma=E_{\mathrm{b}} / N_{0}$ denotes the SNR with $E_{\mathrm{b}}$ bit energy and $N_{0}$ energy of the AWGN. The BER of $M$-QAM for $\gamma$ and averaged for $M$ can be calculated as

$$
P_{\mathrm{b}}(\gamma)=\frac{1}{\log _{2}(\sqrt{M})} \sum_{l=1}^{\log _{2}(\sqrt{M})} P_{\mathrm{b}}(\gamma, l)
$$

In order to embed interference effects into the SINR let us extend $\gamma$ as

$$
\gamma=\frac{\xi \overline{s_{0}} T_{\mathrm{b}}}{N_{0}+I}
$$

In which $T_{b}$ and $I$ are representing the bit time interval and the received energy of the interference. We have also expressed the randomly fluctuating SINR by the $\xi$ random variable, influencing the received $s_{0}$ power as $s_{0}=\overline{s_{0}} \xi$, with the $\overline{s_{0}}$ the average received power. From (4) and after a transformation of the random variables we get the pdf of $\xi$ as

$$
f_{\Xi}(\xi)=\left(\frac{\overline{s_{0}} T_{\mathrm{b}}}{N_{0}+I}\right)^{\alpha} \frac{\xi^{\alpha-1} e^{-\xi} \overline{\frac{\bar{s}_{0} T_{\mathrm{b}}}{\left(N_{0}+I\right) \theta}}}{\Gamma(\alpha) \theta^{\alpha}}
$$


After that the average BER based on (12) can be calculated as $P_{b}\left(\overline{s_{0}}\right)=\int_{0}^{\infty} P_{b}\left(\overline{s_{0}} \mid \xi\right) f_{\Xi}(\xi) d \xi$. For $\phi$, defined in (2) we give the extension $\phi=N_{R} \frac{s_{0} T_{b}}{N_{0}+I}$. We know, that $\theta=C_{\text {corr }} N_{R} \frac{s_{0} T_{b}}{N_{0}+I}$. After that, we get the expression for the average BER as

$$
\begin{aligned}
& P_{b}\left(\overline{s_{0}}, I\right)=\frac{\ln (2)}{\Gamma(\alpha)\left(C_{\text {corr }} N_{R}\right)^{\alpha} \ln (M)} \times \\
& \left.\times \sum_{l=1}^{\log _{2} \sqrt{M}} \sum_{i=0}^{\left(1-2^{-l}\right) \sqrt{M}-1}(-1) \sqrt{\frac{i 2^{l-1}}{\sqrt{M}}}\right\rfloor\left(2^{l-1}-\left\lfloor\frac{i 2^{l-1}}{\sqrt{M}}+\frac{1}{2}\right\rfloor \int_{0}^{\infty} \operatorname{erfc}\left(\sqrt{\xi} \sqrt{\frac{\overline{s_{0}} T_{b}}{N_{0}+I}} \sqrt{\frac{3 \log _{2}(M)}{2(M-1)}}(2 i+1)\right) \xi^{\alpha-1} e^{-\frac{\xi}{C_{\text {cor }} N_{R}}} d \xi .\right.
\end{aligned}
$$

After calculating the integration in closed form for $\alpha>0$, i.e. for $N_{R} \geq N_{T}-1$, the overall average BER can be expressed as

$$
\begin{aligned}
& P_{b}\left(\overline{s_{0}}, I\right)=\frac{\Gamma(2 \alpha) 2^{1-2 \alpha} \ln (2)}{\Gamma(\alpha)\left(C_{\mathrm{corr}} N_{R}\right)^{\alpha} \ln (M)} \sum_{l=1}^{\log _{2} \sqrt{M}} \sum_{i=0}^{\left(1-2^{-l}\right) \sqrt{M}-1}(-1)\left[\frac{i 2^{l-1}}{\sqrt{M}}\right\rfloor\left(2^{l-1}-\left\lfloor\frac{i \times 2^{l-1}}{\sqrt{M}}+\frac{1}{2}\right\rfloor\right) \times \\
& \times\left(\sqrt{\overline{\frac{\bar{s}_{0} T_{b}}{N_{0}+I}}}(2 i+1) C_{1}\right)^{-2 \alpha}{ }_{2} F_{1}\left(a, \frac{1}{2}+a ; 1+a ;-\frac{1}{\frac{\overline{s_{0} T_{b}}}{N_{0}+I} C_{1}^{2}(2 i+1)^{2} C_{\text {corr }} N_{R}}\right),
\end{aligned}
$$

with $C_{1}=\sqrt{\left(3 \log _{2}(M)\right) /(2(M-1))}$, and ${ }_{2} F_{1}(, ; ;)$ representing the regularized hypergeometric function.

\section{Spatial Correlation Model Applied to LTE}

Along the propagation paths between the TX and RX antennas, the channel gain values are spatially correlated [11], (eq. (8)), characterized by the $\mathbf{R}_{\text {MIMO }}=\mathbf{R}_{\mathrm{R}} \otimes \mathbf{R}_{\mathrm{T}}$ spatial correlation matrix, defined by the $\mathbf{R}_{\mathrm{R}}$ transmit- and $\mathbf{R}_{\mathrm{T}}$ receive- correlation matrices (downlink (DL) is assumed), where $\otimes$ represents the Kronecker-product. We set $\mathbf{R}_{\mathrm{T}}$ and $\mathbf{R}_{\mathrm{R}}$ according to the LTE specification [12], for 1, 2 and 4 (TX or $\mathrm{RX)}$ antennas as

$$
\begin{gathered}
\mathbf{R}_{\mathrm{T}}=1, \\
\mathbf{R}_{\mathrm{T}}=\left(\begin{array}{cc}
1 & \alpha \\
\alpha^{*} & 1
\end{array}\right) \\
\mathbf{R}_{\mathrm{T}}=\left(\begin{array}{cccc}
1 & \alpha^{1 / 9} & \alpha^{4 / 9} & \alpha \\
\left(\alpha^{1 / 9}\right)^{*} & 1 & \alpha^{1 / 9} & \alpha^{4 / 9} \\
\left(\alpha^{4 / 9}\right)^{*} & \left(\alpha^{1 / 9}\right)^{*} & 1 & \alpha^{1 / 9} \\
\alpha^{*} & \left(\alpha^{4 / 9}\right)^{*} & \left(\alpha^{1 / 9}\right)^{*} & 1
\end{array}\right) .
\end{gathered}
$$


Note that for $\mathbf{R}_{\mathrm{R}}$ the same forms has been defined with the change of $\alpha \rightarrow \beta$. Unfortunately [12] does not contain any definition for 6 and 8 antennas. To constitute an approximate solution for these cases, we fit a polynomial of degree 2 to the exponents of $\alpha$ and $\beta$ that fits the exponents best in a least-squares sense. After that we get $f(x)=0.111 x^{2}-0.222 x+0.111$. Towards getting the values of $\alpha$ or $\beta$ for the entries of $\mathbf{R}_{\mathrm{T}}^{\left(1, N_{\mathrm{T}}\right)}$ and $\mathbf{R}_{\mathrm{T}}^{\left(N_{\mathrm{T}}, 1\right)}$ or $\mathbf{R}_{\mathrm{R}}^{\left(1, N_{\mathrm{R}}\right)}$ and $\mathbf{R}_{\mathrm{R}}^{\left(N_{\mathrm{R}}, 1\right)}$ respectively, we perform the normalization $F_{N_{\mathrm{T}}}(x)=F_{N_{R}}(x)=\frac{f(x)}{f\left(N_{T}\right)}$. After that, we get two vectors, being the exponents of $\alpha$ and $\beta$ for 6 and 8 antenna cases, i.e.

$$
\begin{gathered}
\mathbf{x}^{(6)}=(0,0.04,0.16,0.36,0.64,1) . \\
\mathbf{x}^{(8)}=(0,0.0204,0.0816,0.1837,0.3265,0.5102,0.7347,1) .
\end{gathered}
$$

It can be shown that $\mathbf{R}_{\mathrm{R}}$ for 6 antennas can be given with $\beta=\alpha$, and the 8 antenna $\mathbf{R}_{\mathrm{T}}$ and $\mathbf{R}_{\mathrm{R}}$ cases can be also calculated according to $\mathbf{x}^{(8)}$. Let us calculate $\theta(\phi)=C_{\text {corr }} \phi$. We assume identity matrix both for $\mathbf{P}$ and $\mathbf{V}$ and $N_{\mathrm{R}}=N_{\mathrm{T}}$. Table 1 shows calculation results according to $\theta=\frac{1 / N_{\mathrm{R}}}{\left[\mathbf{R}^{-1}\right]_{n, n}}$.

Table 1. Calculation Results for $\theta(\phi)$ at Different MIMO Sizes and Correlation Scenarios

\begin{tabular}{llr}
\hline \hline MIMO size & correlation scenario & $\theta=C_{\text {corr }} \cdot \phi$ \\
\hline $2 \times 2$ & low & $1 / 2 \phi$ \\
$2 \times 2$ & med & $91 / 200 \phi$ \\
$2 \times 2$ & high & $19 / 200 \phi$ \\
\hline \hline $4 \times 4$ & low & $1 / 4 \phi$ \\
$4 \times 4$ & med & $0.0134 \phi$ \\
$4 \times 4$ & high & $1.7952 \times 10^{-5} \phi$ \\
\hline \hline $6 \times 6$ & low & $1 / 6 \phi$ \\
$6 \times 6$ & med & $8.2235 \times 10^{-5} \phi$ \\
$6 \times 6$ & high & $7.9889 \times 10^{-10} \phi$ \\
\hline \hline
\end{tabular}

\section{Calculation Results}

This section contains the BER calculation results for different correlation and interference scenarios.

\subsection{BER Results for M-QAM with Average Interference Level}

Fig. 1 contains BER curves for different $M$ modulation levels and SIR=10; $30 \mathrm{~dB}$ for medium correlated MIMO channel, as it was defined in Table 1 for LTE.

Fig. 2 illustrates the results of BER calculations for different modulation levels with SIR=30 $\mathrm{dB}$ for three different correlation scenarios. Both figures are confirming our expectations in terms of the different SIR and correlation scenarios. These calculations show that we are able to calculate exactly the BER values with considering the symbol mapping. 


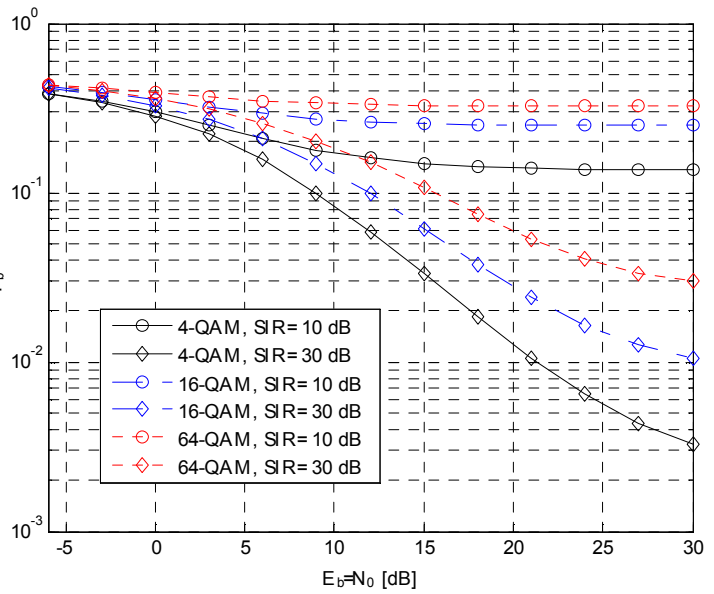

Fig. 1. BER vs. SNR by different SIR values for multiple $M$ levels, assuming medium correlated $2 \times 2$ MIMO.

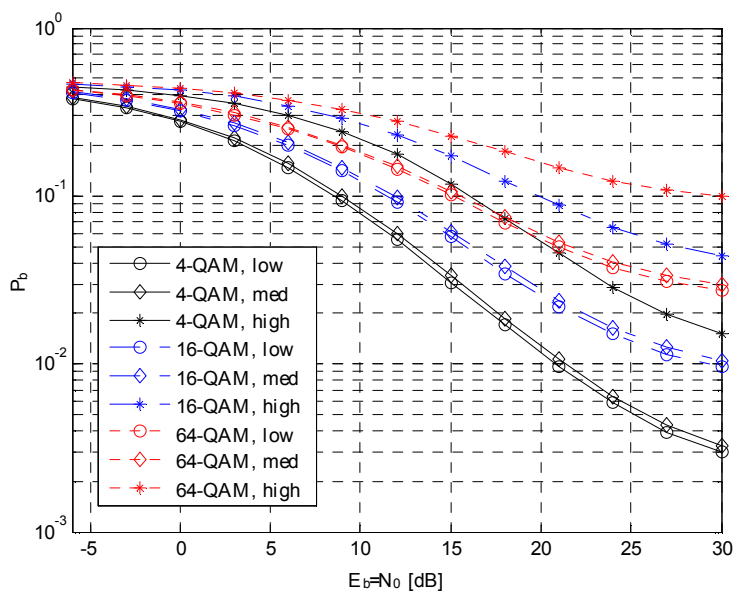

Fig. 2. BER vs. SNR by 4-, 16-, 64-QAM and different correlation scenarios, SIR=30 dB and $2 \times 2$ MIMO.

\subsection{OFDM Interference with Frequency Hopping}

Let us extend the received interference energy $I$, introduced in (8) by a stochastic behavior according to [13] and [14], considering OFDM useful signals and interferers with $\operatorname{sinc}(x)=\sin (x) / x$ spectral shapes. The received interference energy $[\mathrm{J}=\mathrm{W} / \mathrm{Hz}]$ has been considered as $I=\sum_{k=1}^{K} I_{k}=\sum_{k=1}^{K} s_{k} \times v\left(\Delta f_{k}\right)$, with the $I_{k}$ received interference energy and $s_{k}$ received power from interferer $k$. The value of function $v\left(\Delta f_{k}\right)$ represents an amount with dimension of [1/Hz], which is dependent on the spectral overlap between the useful signal and interfering signal $k$. For OFDM interference model $v\left(\Delta f_{k}\right)$ can be given as

$$
v\left(\Delta f_{k}\right)=v\left(l_{k} \times \Delta f_{\mathrm{c}}\right)=\left\{\begin{array}{ll}
\frac{1}{l_{k}^{2} \pi^{2}} \frac{1}{\Delta f_{\mathrm{c}}}, & l_{k} \neq 0 \\
\frac{2}{3} \frac{1}{\Delta f_{\mathrm{c}}}, & l_{k}=0
\end{array},\right.
$$

For $l_{k} \in \mathbb{Z}$, with $\Delta f_{c}$ subcarrier spacing, according to [13]. We assume that the spectral distances between the $f_{\mathrm{c}}$ and $f_{k}$ center frequencies of the useful- and interference signals can be always given with $\Delta f_{k}=\left|f_{\mathrm{c}}-f_{k}\right|=l_{k} 2 \Delta f_{\mathrm{c}}$. We consider $l_{k}$ as a uniform $\mathrm{RV}$ on $l \in\{1,2, \ldots, N\}$, since $f_{k}$ can be located at 
both sides of $f_{\mathrm{c}}$. In addition, let $s_{k}=s_{1}$ and $l_{k}=l_{1}, \forall k$. After that the BER according (11) will be conditional to $l_{k}$ and $K$. Using $I=\sum_{k=1}^{K} s_{k} v\left(\Delta f_{k}\right)$ and (14), the received interference can be expressed as

$$
I\left(l_{1}, K\right)=\frac{s_{1}}{\pi^{2} \Delta f_{c}} \frac{K}{l_{1}^{2}}
$$

Thenceforward, based on (11) we will perform the average BER calculation with

$$
P_{b}\left(\overline{s_{0}}, K\right)=\mathbb{E}\left[P_{b}\left(\overline{s_{0}} \mid l_{1}, K\right)\right]=\frac{1}{N} \sum_{l_{1}=1}^{N} P_{b}\left(\overline{s_{0}} \mid l_{1}, K\right) .
$$

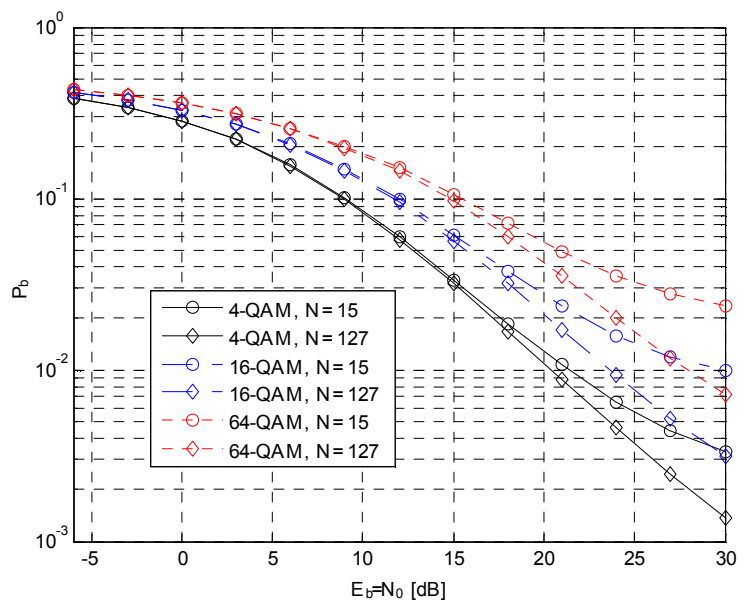

Fig. 3. BER vs. SNR by 4-, 16- and 64-QAM for $N=15,127$ and $2 \times 2$ MIMO.

Fig. 3 shows the BER results of our investigation in terms of modifying the interval of the frequency hopping, i.e. the $N$ multiplication factor of $\Delta f_{\mathrm{c}}$. With increasing $N$, the $f_{k}$ frequencies of the interferers may be located to larger spectral distances from $f_{\mathrm{c}}$, resulting lower BER values. We can observe this effect for different modulation levels for single interferer $(K=1)$.

Fig. 4 contains the results for the same FH interference model with $K=1$ and $K=10$. It is not surprising that a larger number of interferers results higher BER values.

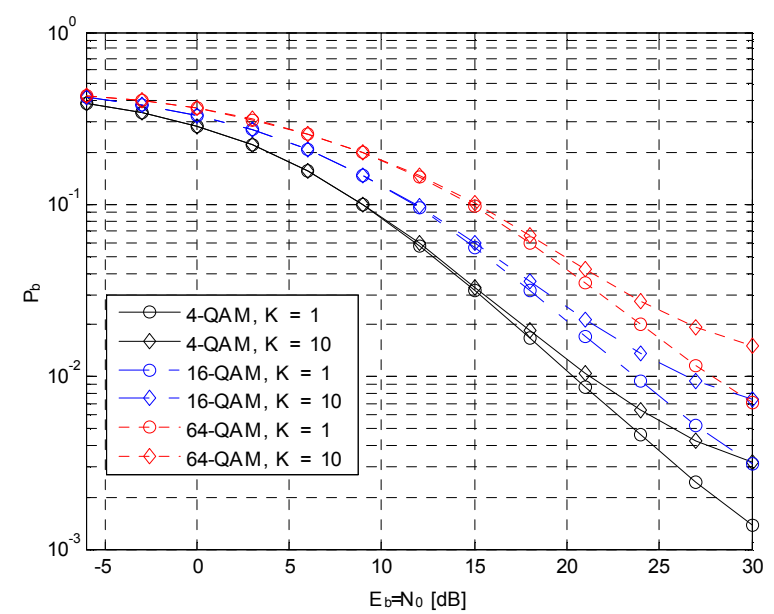

Fig. 4. BER vs. SNR by 4-, 16- and 64-QAM for $K=1,10$ and $2 \times 2$ MIMO. 
We note that the stochastic extension of the received $I$ interference energy above can be applied for different discrete random distributions for $l_{k}$ or even for (distance dependent) instantaneous received $s_{k}$ values, by introducing different pathloss models etc.

\section{Acknowledgment}

The work reported in the paper has been developed in the framework of the 'Intelligent cellular network: A Two-Tier Cellular Architecture with Robust Interference Capability and Optimal Resource Management' project. This project is supported by the grant TÉT\_10-1-2011-0266.

\section{References}

[1] Hanzo, L., Haas, H., Imre, S., O’brien, D., Rupp, M., \& Gyongyosi, L. (May 2012). Wireless myths, realities, and futures: From 3G/4G to optical and quantum wireless. Proceedings of the IEEE: Vol. 100, Special Centennial Issue (pp. 1853-1888).

[2] Nakajima, A., Garg, D., \& Adachi, F. (2004). Turbo coded MIMO multiplexing with iterative adaptive soft parallel interference cancellation. Proceedings of IEEE 60th Vehicular Technology Conference, VTC-Fall: Vol. 2 (pp. 1410-1414).

[3] Gritsch, G., Weinrichter, H., \& Rupp, M. (May 2004). A union bound of the bit error ratio for data transmission over correlated wireless MIMO channels. Proceedings of IEEE International Conference on Acoustics, Speech, and Signal Processing: Vol. 4 (pp. 405-408).

[4] Kim, I. M. (Aug. 2006). Exact BER analysis of OSTBCs in spatially correlated MIMO channels. IEEE Transactions on Communications, 54(8), 1365-1373.

[5] Kammoun, A., Kharouf, M., Hachem, W., \& Najim, J. (Oct. 2009). BER and outage probability approximations for LMMSE detectors on correlated MIMO channels. IEEE Transactions on Information Theory, 55(10), 4386-4397.

[6] Kim, N., \& Park, H. (July 2009). Bit error performance of convolutional coded MIMO system with Linear MMSE receiver. IEEE Transactions on Wireless Communications, 8(7), 3420-3424.

[7] Mohammadnia-Avval, M., Snow, C., \& Lampe, L. (June 2010). Error-rate analysis for bit-loaded coded MIMO-OFDM. IEEE Transactions on Vehicular Technology, 59(5), 2340-2351.

[8] Armada, A. G., Hong, L., \& Lozano, A. (2009). Bit loading for MIMO with statistical channel information at the transmitter and ZF receivers. Proceedings of IEEE International Conference on Communications (pp. 1-5).

[9] Li, P., Paul, D., Narasimhan, R., \& Cioffi, J. (Jan. 2006). On the distribution of SINR for the MMSE MIMO receiver and performance analysis. IEEE Transactions on Information Theory, 52(1), 271-286.

[10] Cho, K., \& Yoon, D. (July 2002). On the general BER expression of One and two-dimensional amplitude modulations. IEEE Transactions on Communications, 50(7), 1074-1080.

[11] Kermoal, J., Schumacher, L., Pedersen, K., Mogensen, P., \& Frederiksen, F. (Aug. 2002). A stochastic MIMO radio channel model with experimental validation. IEEE Journal on Selected Areas in Communications, 20(6), 1211-1226.

[12] ETSI. (2012). Network, Evolved Universal Terrestrial Radio Access (E-UTRA); User Equipment (UE) Radio Transmission and Reception. 3GPP Technical Specification 36.101 Version 11.0.0.

[13] Mraz, A. (2013). Modeling and Optimization of Advanced Wireless Networks. Lambert Academic Publishing.

[14] Mraz, A., \& Pap, L. (2012). General interference analysis of M-QAM and M-PSK wireless communications. Wireless Networks, 19(3), 331-344. 


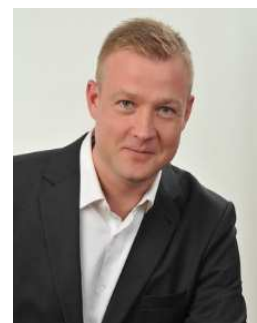

Albert Mráz was born in 1979. He had received the MSc and PhD degrees in electrical engineering from Budapest University of Technology and Economics (BME), in Hungary in 2005 and 2013 respectively. His research interests include mainly PHY and MAC layer modeling and resource management of broadband wireless networks (involving OFDM, OFDMA, MIMO, scheduling, interference management etc.). He is currently an assistant research fellow at the Department of Networked Systems and Services at BME, Budapest, Hungary.

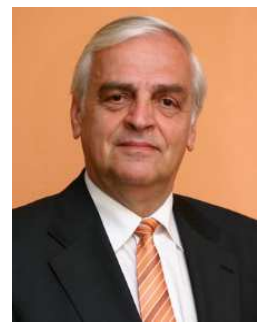

László Pap was born in 1943. He graduated from Budapest University of Technology and Economics (BME), Hungary, Faculty of Electrical Engineering. He is a full member of the Hungarian Academy of Sciences. His main fields of the research are electronic systems, nonlinear circuits, synchronization systems, modulation and coding, spread spectrum systems, ATM, CDMA, multiuser detection and mobile communication systems. Professor Pap had been the head of the Department of Telecommunications (later Department of Networked Systems and Services), Budapest University of Technology. 